\title{
JUVENILE CHURG-STRAUSS SYNDROME - CASE REPORT
}

\section{Ricardo Amaro Noleto Araujo1,2,*, Mirela Bianchezi Fernandes², Giovanna Garcia Stabile², Alisson Lisboa Cordeiro², Raquel de Nardo Claudino², Samy Simantob Fridman², Sany Tomomi de Almeida Rocha Arita²}

1. Hospital Santa Marcelina, São Paulo (SP), Brazil. 2. Faculdade Santa Marcelina, São Paulo (SP), Brazil.

*Corresponding author: ricardoreumatologista@gmail.com

\section{BACKGROUND}

Eosinophilic granulomatosis with polyangiitis (EGPA), or Churg-Strauss syndrome, is a systemic vasculitis characterized by asthma and eosinophilia, affecting men and women at a ratio of 2:1, average incidence between 30-40 years. In children the incidence is unknown yet because of the lack ok studies about EGPA in pediatrics. The characteristics are asthma, pulmonary infiltrates, eosinophilia, heart failure, kidney disease and peripheral neuropathy.

\section{CASE REPORT}

R.A.Q., 14 years old, male, previously healthy, reported shortness of breath, asthenia for 2 months, asthma and pain in the lower limbs with severe intensity. Seeking medical assistance in August/2017, reporting intensive epigastric pain, bronchospasm and paresthesia in the inferior members. Upon admission, the patient developed cyanosis in the right midfoot, with progression to necrosis (Fig. 1). Tests results indicate eosinophilia of $14,285 / \mathrm{mm}^{3}$ ( $50 \%$ of the total), an increase in ESR, PCR and negative ANCA. Arteriography of the lower limbs resulted occlusion of the anterior and posterior tibial artery (Fig. 2). Methylprednisolone $1.0 \mathrm{~g}$ was administered for 3 days associated with $1.0 \mathrm{~g}$ cyclophosphamide. Despite treatment, he required right transmetatarsal amputation. The Birmingham vasculitis activity score (BVAS) was 29 . On the outpatient care, he received monthly cyclophosphamide associated with $1.0 \mathrm{mg} / \mathrm{kg}$ oral corticosteroids, which evolved with an adequate response to treatment. In February/2018, he was hospitalized for chest pain associated with hypertension and troponin elevation, developing severe bronchospasm. Then referred to the ICU for mechanical ventilation. Laboratory tests results $14,671 / \mathrm{mm}^{3}$ eosinophilia ( $65 \%$ of the total), increased troponin and BNP, with myocardial MRI showing signs of acute myocarditis. It was suggested switching treatment to rituximab or mepolizumab; however, due to unavailability, a new induction with methylprednisolone $1.0 \mathrm{~g}$ for 3 days associated with cyclophosphamide $1.2 \mathrm{~g}$ in the Cyclops regimen was performed, at the time BVAS-13. The second response was excellent. Corticosteroids withdrawal occurred in 9 months from February to November 2018. In July 2019, he started maintenance with azathioprine $2.6 \mathrm{mg} / \mathrm{kg} /$ day with 18 months of follow-up in BVAS remission (0).

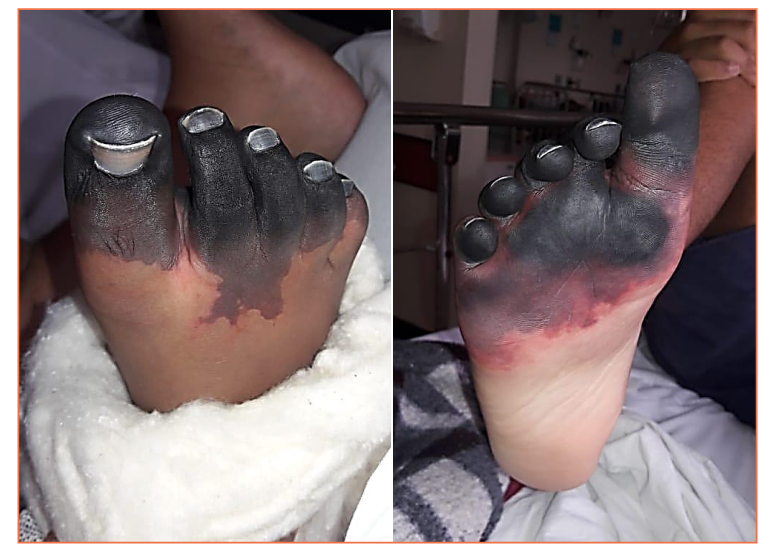

Figure 1. Photo of vascular necrosis of right middle foot after 3 days of hospitalization. 


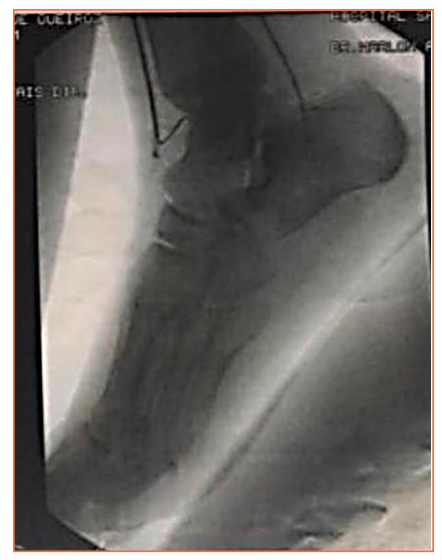

Figure 2. Arteriography of the right foot showing occlusion of the anterior and posterior tibial artery.

\section{CONCLUSION}

The case described has a low incidence in the juvenile age group and aims to outline the prodromal phase, with pneumonia before EGPA, followed by eosinophilia associated with the vasculitic phase and manifestation of cyanosis and necrosis in the midfoot. Evolving to eosinophilic myocarditis. It should be noted that when the condition worsened, prednisone was administered $0.15 \mathrm{mg} / \mathrm{kg} /$ day and had already performed 7 pulses of cyclophosphamide. Other therapies consisting of rituximab and mepolizumab need studies in this population. This report reinforces the need for further studies to treat this pathology. 